\title{
BioéthiqueOnline
}

\section{Faire dialoguer les cultures : rencontre entre la bioéthique et l'art contemporain}

\section{Marianne Cloutier}

Volume 5, 2016

URI : https://id.erudit.org/iderudit/1044288ar

DOI : https://doi.org/10.7202/1044288ar

Aller au sommaire du numéro

Éditeur(s)

BioéthiqueOnline

ISSN

1923-2799 (numérique)

Découvrir la revue

Citer cet article

Cloutier, M. (2016). Faire dialoguer les cultures : rencontre entre la bioéthique et l'art contemporain. BioéthiqueOnline, 5. https://doi.org/10.7202/1044288ar
Résumé de l'article

Cet essai aborde la rencontre entre les chercheurs en bioéthique et les artistes qui ont participé au projet Art + Bioéthique. La co-commissaire de l'exposition liée à ce projet présente ici la manière dont chaque artiste problématise un champ de recherche spécifique et en propose une interprétation personnelle à travers son oeuvre. 


\title{
Faire dialoguer les cultures : rencontre entre la bioéthique et l'art contemporain
}

\author{
COMMENTAIRE CRITIQUE / CRITICAL COMMENTARY (RÉVISION PAR LES PAIRS / PEER-REVIEWED) \\ Marianne Cloutier ${ }^{1}$
}

\author{
Reçu/Received: 29 Mar $2016 \quad$ Publié/Published: 16 Sept 2016 \\ Éditrices/Editors: Lise Levesque \& Aliya Affdal \\ Évaluateurs externes/Peer-Reviewers: Jens Hauser \& Paul Ardenne
}

2016 M Cloutier, Creative Commons Attribution 4.0 International License

\section{Résumé}

Cet essai aborde la rencontre entre les chercheurs en bioéthique et les artistes qui ont participé au projet Art + Bioéthique. La co-commissaire de l'exposition liée à ce projet présente ici la manière dont chaque artiste problématise un champ de recherche spécifique et en propose une interprétation personnelle à travers son œuvre.

\section{Mots clés}

art contemporain, bioéthique, médiation, collaboration artiste-chercheur

\section{Summary}

This essay discusses the encounter between bioethics researchers and artists who have participated in the Art + Bioethique project. The co-curator of the exhibition related to this project presents here the way that each artist problematized a specific area of research and offers a personal interpretation through their work.

\section{Keywords}

contemporary art, bioethics, mediation, artist-researcher collaboration

\section{Responsabilités des évaluateurs externes}

Les évaluations des examinateurs externes sont prises en considération de façon sérieuse par les éditeurs et les auteurs dans la préparation des manuscrits pour publication. Toutefois, être nommé comme examinateur n'indique pas nécessairement l'approbation de ce manuscrit. Les éditeurs de BioéthiqueOnline assument la responsabilité entière de l'acceptation finale et la publication d'un article.

\section{Peer-reviewer responsibilities}

Reviewer evaluations are given serious consideration by the editors and authors in the preparation of manuscripts for publication. Nonetheless, being named as a reviewer does not necessarily denote approval of a manuscript; the editors of BioéthiqueOnline take full responsibility for final acceptance and publication of an article.

Affiliations des auteurs / Author Affiliations

${ }^{1}$ Chercheure postdoctorale, Département de sciences biologiques, Université de Montréal, Montréal, Canada

\section{Correspondance / Correspondence}

Marianne Cloutier, marianne.cloutier.1@umontreal.ca

\section{Remerciements}

L'auteure tient à remercier Jean-Christophe Bélisle-Pipon, Vincent Couture, Maude Laliberté, Catherine Barnabé et Aïda Lorrain pour leur précieuse collaboration tout au long de ce projet. Le stage postdoctoral de Marianne Cloutier est appuyé financièrement par le Fonds de recherche du Québec - Société et culture (FRQ-SC).

\section{Conflit d'intérêts}

Aucun déclaré

\section{Acknowledgements}

The author would like to thank Jean-Christophe BélislePipon, Vincent Couture, Maude Laliberté, Catherine Barnabé and Aïda Lorrain for their precious collaboration throughout the project. The postdoctoral fellowship of Marianne Cloutier is financially supported by the Fonds de recherche du Québec - Société et culture (FRQ-SC).

\section{Conflicts of Interest}

None to declare

\section{De la recherche à la pratique artistique}

Le projet Art + Bioéthique est né de la volonté de trois chercheurs - Jean-Christophe Bélisle-Pipon, Vincent Couture et Maude Laliberté - de faire sortir la recherche universitaire en bioéthique de son cadre strictement académique. Puisque cette branche spécifique de l'éthique propose de réfléchir à la 
vaste question des usages du vivant, elle fait intervenir des chercheurs issus d'une pluralité de domaines et aux formations variées. Selon la perspective disciplinaire qui leur est propre, leur rôle est donc de proposer un cadre théorique permettant de comprendre et d'évaluer les choix, les valeurs ou les normes morales en jeu dans un contexte ou une situation précise. Bien que la bioéthique ait peu à peu imprégné le discours commun au cours des dernières décennies - notamment en raison de la progression fulgurante des technosciences et des biotechnologies dans maintes sphères de notre société - elle en demeure pourtant encore mal connue. Il est vrai d'ailleurs qu'elle peut sembler aride et hermétique, peut-être encore davantage lorsque, dans sa dimension prescriptive, elle est entremêlée au droit pour se faire la gardienne des « bonnes » normes sociales.

Les œuvres qui ont été conçues dans le cadre de l'exposition Art + Bioéthique (février-mars 2016, galerie Espace Projet) - dont nous avons assuré le commissariat conjointement avec Catherine Barnabé - invitent à modéliser divers aspects touchant à notre condition contemporaine et à réfléchir à une panoplie de questions relatives à la bioéthique qui devront, tôt ou tard, être débattues au sein de la société, lorsqu'elles ne le sont pas déjà. Dans certains cas, elles sembleront peut-être s'éloigner quelque peu de ce domaine, mais les problèmes philosophiques ou les interrogations qui y sont présentés sont pourtant ceux qui ont émergé au cours des échanges des binômes. Créé par un artiste et un chercheur, jumelés selon leurs intérêts de recherche et de création respectifs, chaque binôme a en effet reçu peu de contraintes, mis à part celle d'une série de rencontres échelonnées sur cinq mois et ayant comme point de départ un dialogue axé sur les recherches de chacun. Les œuvres inspirées de ces discussions furent donc présentées en parallèle à des essais produits par les chercheurs. Ainsi, nous présentons ici chacun des binômes ayant participé au projet. Pour chacun d'eux, nous tenterons de résumer les objectifs des recherches du bioéthicien et d'expliquer de quelle manière ils sont liés à l'œuvre qu'a produite l'artiste pour l'exposition. De plus, nous proposerons des pistes de lecture et d'interprétation de celle-ci pour mettre en lumière les éventuels liens établis entre l'univers de la recherche et celui de la création.

\section{La relation de l'humain à l'animal}

C'est autour du concept de responsabilité morale des médecins en regard de leurs patients animaux que gravitent les recherches de Dominick Rathwell-Deault. Celle-ci a été jumelée avec Audrey Kinkead, dont la pratique artistique a comme point nodal une préoccupation pour l'animal. Elles ont réfléchi ensemble à la relation que nos sociétés entretiennent avec celui-ci, notamment à travers des problèmes tels que l'euthanasie de convenance, qui traduit une conception utilitariste de la bête, encore très répandue aujourd'hui. Le biocentrisme fut également un des sujets discutés qui a influencé la création de Kinkead. En effet, selon ce courant de l'éthique environnementale, la vie en soi est considérée comme ayant une valeur intrinsèque et par le fait même, tous les êtres vivants sont dignes du même respect.

Comme une voie permettant de s'éloigner d'une vision des rapports homme-animal purement anthropocentrique, Kinkead explore cette notion et la matérialise dans son travail par l'intégration sociale des animaux dans l'espace de la ville. Selon elle, une véritable intégration ne se limite pas à l'acceptation des espèces domestiques élues comme compagnons de l'humain - telles que le chat ou le chien -, mais également à celles considérées comme indésirables, voire parfois nuisibles. Son œuvre, 54 rue DuBalcon [1], qui s'inspire de penseurs tels qu'Elizabeth de Fontenay ou Donna Haraway, cherche ainsi à pousser de l'avant l'idée d'accorder un véritable statut à l'animal au sens large et l'accueille comme un membre de la société à part entière : un co-citoyen [2]. Le titre de l'œuvre fait référence au projet de loi 54 établi en 2015 par la province de Québec. Le but de ce dernier est de chercher à améliorer la situation juridique des animaux, les reconnaissant non plus comme une propriété, mais comme des êtres vivants possédant leur propre sensibilité. Telle une concrétisation de leur reconnaissance sociale, l'artiste officialise la place prise par une famille d'écureuils ayant établi domicile dans le hangar attenant à son appartement. Elle leur installe une boîte aux lettres et une adresse - le 54 - leur accordant symboliquement un statut social. Ainsi, ils ne 
sont non plus considérés comme vermine indésirable, envahisseurs des villes, mais comme de respectables voisins. L'artiste officialise ceci par un bail, témoignage d'un contrat social, où l'animal apposera sa propre signature, la trace de sa griffe.

\section{Le vécu du sujet face aux nanotechnologies}

Avec le regard du sociologue, Mathieu Noury s'intéresse depuis plusieurs années aux enjeux éthiques de l'application clinique de la nanomédecine et de la médecine régénératrice. Au-delà de l'aspect fascinant de la prise en charge de la maladie - du diagnostic au traitement - rendue possible par les technologies moléculaires, le problème majeur de cette nouvelle approche biomédicale est, selon lui, la conception du corps et de l'individu qu'elle véhicule : une conception objectivée, un combat contre la maladie dissimulant le fait que derrière ce corps se trouve un être singulier, un individu. C'est pour cette raison que le chercheur et l'artiste Stéphanie Coleman ont choisi de concert le titre Na-no-body [3,4], référence évidente aux nanotechnologies, mais aussi à l'occultation du corps vécu et sensible.

C'est cette problématique spécifique que Coleman a cherché à illustrer par son installation. En effet, elle crée ici un imaginaire fantasmatique autour des nanotechnologies, une interprétation personnelle peuplée de motifs et d'objets énigmatiques, qui traduisent l'aspect mystérieux que représentent les nanosciences pour le profane. Telle une tapisserie baroque finement dessinée, l'œuvre présente des motifs qui semblent issus de l'hybridation de végétaux et de cellules vivantes ou d'autres composantes biologiques indéterminées. Ces formes représentées donnent par moment l'impression de sortir de l'espace pour se matérialiser à travers de petits éléments sculpturaux qui adoptent une iconographie similaire. Comme si le paysage plat et impersonnel de la tapisserie prenait corps, cette continuité entre le dessin et l'objet sculpté donne à penser que l'individu cherche ainsi à se déployer dans l'espace, à témoigner de sa présence réprimée. Et pourtant, dans ce décor noir et blanc, tout demeure fixe, sans couleur et sans vie, loin du corps de chair palpitant. Tout demeure indifférencié, sans identité certaine, des éléments biologiques aussi indéterminés qu'interchangeables, qui pourraient finalement provenir de n'importe quel être.

Dans une série de boîtes de Petri intégrées à l'installation reposent de petits objets aux formes variées, mais similaires, peut-être d'infimes parties de corps qu'on aurait retirées lors d'une intervention. Ou peut-être s'agit-il plutôt de nanostructures aux pouvoirs indéterminés, grossies maintes fois par l'œil du microscope. Quant aux minuscules lumières incrustées à la tapisserie et que le spectateur pourra activer au toucher, elles mettent l'accent sur l'aspect " technologique » de cette médecine moléculaire. Elles réitèrent également le problème de son rapport au patient et ramènent symboliquement une composante sensible à travers l'acte du toucher. Ce toucher demeure cependant froid et sans impact réel, paradoxe qui illustre certainement le défi bioéthique propre aux nanosciences, celui de ramener un peu plus d'humanité et d'empathie dans cette technologie de pointe : il invite à penser une éthique du soin dans le rapport établit au patient.

\section{Explorer les " émotions négatives ॥ face au handicap}

Formé au droit et à la bioéthique, Jonas-Sébastien Beaudry s'intéresse dans le cadre de ses recherches à l'égalité et à l'inclusion sociale des personnes handicapées. Au fil de ses échanges avec l'artiste bordelais Mael Le Mée, plusieurs sujets qui lui sont chers viennent teinter la discussion et influencer concrètement le projet en devenir de ce dernier. Ce sont spécifiquement les questions des émotions négatives, de la normalisation ainsi que de la conceptualisation du sujet à travers les discours moraux, politiques et légaux qui inspireront l'œuvre créée pour Art + Bioéthique. En collaboration avec la danseuse et chorégraphe montréalaise France Geoffroy, dont l'une des particularités est d'être tétraplégique, Le Mée propose France-e(s)t-Mael, un "duo siamois transatlantique » qui prend forme à travers une performance en deux temps, partiellement documentée par une vidéo. Dans celle se déroulant à Bordeaux, Le Mée prend place dans un fauteuil 
roulant électrique et effectue un parcours à travers la ville, expérimentant un point de vue pour lui inhabituel. Ce point de vue, c'est pourtant celui vécu au quotidien par Geoffroy. Ainsi, il peut témoigner de certaines contraintes liées notamment aux espaces non adaptés ou à la complexité que prennent certains gestes à la base pourtant anodins.

Au cours de la performance, Geoffroy contrôle à distance des électrodes placées sur un des avantbras de l'artiste et commande ainsi certains de ses mouvements. Ainsi, le dispositif technologique permet à Le Mée de léguer une partie du pouvoir sur les mouvements de son corps, mais également sur ses interactions sociales. En effet, pour ce duo "siamois", la danseuse habite avec lui son corps puisque sa tête s'y trouve juxtaposée via un iPad qui y a été préalablement fixé. Par la téléprésence, elle pourra donc non seulement dialoguer avec l'artiste, mais aussi interagir avec les gens croisés au fil de leur parcours. La performance inverse, Mael-e(s)t-France [5], a été réalisée à Montréal à la fin février 2016, via le même dispositif, mais ces alors Geoffroy qui partagera en partie son corps.

Pour Mael Le Mée, ce projet se veut avant tout une exploration du concept de monstre tel que l'a formulé Michel Foucault dans Les anormaux, cours au Collège de France. 1974-75 soit " celui qui combine l'interdit et l'impossible » [6]. En effet le projet s'intéresse aux deux dimensions abordées par Foucault : son sens biologique et son sens juridique. D'abord, l'être siamois technologique est en soi un corps improbable qui explore cette complexité de la définition du monstrueux. Mais c'est aussi parce qu'il défie la loi juridique - par son essence et sa configuration même - qu'il s'y inscrit. En effet, France-e(s)t-Mael / Mael-e(s)t-France imagine un être transatlantique qui se trouve à la fois dans deux pays différents, qui existe ici et ailleurs, et qui peut ainsi agir dans deux systèmes législatifs différents. Et puisque, selon le lieu de la performance, les deux êtres qui agissent en ce corps prennent en partie le pouvoir sur l'autre, comment s'inscrivent-ils au sein du système législatif de chaque pays? Si par exemple Geoffroy pose un geste répréhensible par la loi alors qu'elle n'est pas en plein contrôle de son corps, mais dirigée via l'électrostimulation, comment le système juridique vat-il transiger avec cet acte? Ce corps hors-norme qui existe à la fois de manière réelle et fantasmée, explore en effet la loi comme une construction langagière, comme l'est aussi la conception du monstre foucaldien.

Si ce projet amène d'emblée une réflexion sur le regard posé sur le corps hors-norme, sur le contraste du vécu et sur la manière dont la société en général traite la différence, il présente toutefois ce corps autre de manière ludique. II peut en effet s'agir d'une manière de contrer ces émotions négatives face au handicap - pitié, peur, colère, etc. - dont discute Beaudry dans son essai " The Anxious Heart of Injustice: Negative Affective Responses to Disabilities » [7]. Loin du pathos, de la souffrance ou de la douleur, ce n'est pas un corps que l'on plaint, mais un corps aux possibilités augmentées par son appareillage technologique et la complicité réunissant Geoffroy et Le Mée. Le partage d'un corps et d'une expérience vécue permet un dialogue double, de nouveaux types d'interactions via le corps de l'autre, et un regard transformé sur le monde. Ainsi, l'artiste propose le hors-norme comme une forme d'empowerment et transcende la notion de limite qui pourrait être associée au corps tétraplégique de Geoffroy en en proposant un sens retourné : c'est par ce corps qu'il pourra virtuellement traverser des milliers de kilomètres à partir de la France et se retrouver là, en sol québécois. C'est grâce à ce corps « siamois », avec lequel il se déplacera, qu'il pourra obtenir cet effet de présence.

Malgré cette symbolique forte, entrecroisée au fait que Geoffroy se livre avec enthousiasme à cette expérimentation et qu'on puisse y lire, comme pour sa pratique de la danse contemporaine, cet acte du corps comme un geste artistique positif, créatif et même activiste, certains recevront peut-être l'œuvre avec un certain malaise. On peut sans doute se demander si ce malaise n'a pas été souhaité par l'artiste. En effet, l'œuvre cherche peut-être volontairement à nous plonger au cœur de l'inconfort afin d'opérer une forme de catharsis, de briser la gêne ressentie face aux représentations du corps handicapé ou hors-norme : nous faire vivre intensément ces émotions négatives pour s'en libérer et 
par le fait même, laisser enfin à ces corps et ces individus, l'espace social qui leur revient, tout en questionnant le nôtre.

\section{Naissance et fragilité de la vie}

Dans le cadre de ses recherches en droit, Jean-Frédéric Ménard s'intéresse aux questions bioéthiques spécifiques aux soins intensifs néonataux, plus particulièrement à l'émergence et à la négociation des normes encadrant la prise de décision éthique et juridique dans ce contexte particulier. C'est en étudiant les processus sociaux qui régissent le fonctionnement des unités de soins intensifs qu'il mène son travail de chercheur. Comme il l'explique dans son essai « Cobayes de la relève : l'artiste et le chercheur à la rencontre de l'expérience » [8], il y est témoin d'expériences vécues par ces équipes confrontées chaque jour à l'essence même de l'éthique, c'est-à-dire à la question " comment faire pour bien faire? ». En effet, les professionnels dédiés aux soins néonataux sont perpétuellement confrontés à des décisions complexes, où la fragilité de la vie est exacerbée, ils vivent des expériences où la vie d'un être encore à naître ou ayant à peine vu le jour est en jeu.

Cette importance primordiale de l'expérience vécue et de la notion de fragilité trouvent écho dans l'ensemble du travail de l'artiste Arkadi Lavoie Lachapelle, et encore plus spécifiquement dans Jour de fête! [9], l'œuvre qu'elle expose dans le cadre de Art + Bioéthique. II s'agit en fait d'une réappropriation d'une photo d'archive, prise lors d'une naissance en 1989 par Isabelle Brabant : une activiste et pionnière ayant beaucoup milité pour la reconnaissance des sages-femmes au Québec, ainsi que pour la revendication de naissances moins médicalisées et plus naturelles. Même si cette profession demeure encore aujourd'hui dans un réseau en marge à la structure médicale, l'œuvre rappelle l'immense pas qui a été franchi depuis la fin des années 1980 quant à leur travail, notamment avec la mise en place des maisons de naissance.

L'artiste est intervenue de différentes manières sur l'objet photo initial. Pour transformer cette archive en œuvre et ajouter à son ready-made un regard autre, Lavoie-Lachapelle a d'abord agrandi maintes fois l'image, la rendant beaucoup plus spectaculaire et mettant ainsi en évidence certains détails auparavant discrets. Elle y ajoute également un cadre, affirmant son statut d'œuvre, et surtout, elle opère une rotation de 180 degrés et présente l'image à l'envers. Pour l'artiste, cet acte de médiation est primordial et transforme complètement notre manière de visualiser et de comprendre la scène. En effet, l'image originale donne surtout à voir le corps de la mère en plein travail - d'ailleurs dans une posture d'accouchement vraiment inhabituelle pour l'époque - et l'acte médical que s'apprête à opérer le médecin, instrument à la main. On ressent l'urgence du moment et son aspect tragique : le bébé semble mal en point, souffrant, trop pâle pour que tout soit normal. De cette puissante image se dégage une sorte de violence qui la rend difficilement soutenable du regard.

Lorsque retournée, comme la présente l'artiste, notre perception des corps se transforme complètement : le médecin semble être en contre-plongée et les autres individus se confondent à travers le camaïeu de vert des vêtements et des draps d'hôpital. Quant au visage du bébé, il n'est plus à l'envers et devient automatiquement le point focal de l'image. C'est d'ailleurs étrangement le seul visage perceptible dans l'image. Par ce retournement, l'artiste met encore davantage l'accent sur les diverses formes de liminalité à l'œuvre dans la scène : ce moment de passage du statut de fœtus à celui de nouveau-né, d'être au monde; le passage de l'intérieur du corps de la mère à l'univers extérieur; mais aussi dans ce cas précis, l'entre-deux crucial entre la vie et la mort, ce moment critique où tout peut basculer. Ce Jour de fête! sera-t-il dans son issue, un moment heureux, ou au contraire tragique, tout semble reposer sur les gestes et les décisions qui seront prises par le corps médical. L'œuvre rappelle ainsi le poids qui pèse sur les équipes en fonction et la fragilité de la vie en jeu à travers les enjeux de pouvoir sous-jacents. Ce petit visage au centre de l'image interroge aussi qui est le véritable sujet en jeu dans les politiques de néonatalité alors que le politique et l'éthique s'entremêlent. 


\section{Le SOPK et la revendication de la différence}

Dans le cadre de sa formation en bioéthique, Victoria Doudenkova s'intéresse par ses recherches aux enjeux relatifs au soin du syndrome des ovaires polykystiques (SOPK ou en anglais PCOS). Un des désordres endocriniens les plus communs chez les femmes en âge de procréer, ce désordre affecte environ une femme sur dix. Comme l'explique la chercheuse dans son essai « La bioéthique, l'art et le syndrome des ovaires polykystiques: propos impressionniste visant à réhabiliter les corps tabous et les ovaires blâmés » [10], il s'agit d'une des causes majeures d'infertilité et il est lié à des maladies graves telles que les cancers du sein, de l'utérus et des ovaires, des troubles cardiovasculaires et certaines formes de diabète. De surcroit, ses conséquences physiques - hyperandrogénie, perturbation des fonctions sexuelles, graves problèmes de peaux, obésité - affectent également la santé mentale et provoquent dépression et anxiété chez celles qui en sont atteintes. Malgré tout, Doudenkova soulève la rareté des études qui lui sont consacrées, ce qui a pour effet que la maladie demeure mal connue des médecins, les amenant ainsi à en traiter uniquement les symptômes plutôt qu'à guérir la véritable cause.

D'un commun accord avec Grace Stokes, à laquelle elle a été jumelée pour ce projet, l'artiste et la chercheuse décident ici d'adopter l'approche d'une « mise en valeur des failles dans la beauté et la simplicité » [10] plutôt qu'une attitude dénonciatrice. Selon elles, le fait d'observer les faits et de les représenter constitue déjà un pas vers la conscientisation du public et donc vers certaines solutions face à la problématique du SOPK. Ainsi, dans la première partie de son œuvre créée à partir de la sérigraphie et intitulée Constant Beauty Within [11], Stokes se livre à des représentations d'utérus dont les ovaires sont remplacés par des fleurs de prunier mirobolant (cherry plum). Celles-ci réfèrent aux traitements par les élixirs floraux, communément appelés "fleurs de Bach " en référence à son concepteur, le médecin et homéopathe Dr Edward Bach. II s'agit en effet d'un des traitements fréquemment utilisés en médecine alternative et à propos duquel l'artiste et la chercheuse ont longuement échangé. L'impression de douceur et l'aspect inoffensif qui se dégage de l'image peuvent à la fois être perçus comme la critique souvent faite aux médecines alternatives (ou médecines douces) d'être en effet peu agressives, mais aussi plus ésotériques qu'efficaces. Mais pour Stokes, l'aspect délicat ici recherché se veut surtout une célébration de la complexité du corps de la femme qui va bien au-delà de la souffrance et de la douleur associées à la maladie.

De manière générale, la seconde partie de l'œuvre, PCOS as Cacti [11], présente le cactus, comme une traduction littérale de la douleur de l'expérience vécue de la maladie. Les interventions opérées sur chaque plante réfèrent cependant pour Stokes à d'autres aspects de la maladie - les symptômes et les conséquences liées à l'apparence visuelle de la maladie - et de son traitement. Par exemple, l'ajout de peinture dorée symbolise les questions d'ordre financier et le manque de fonds investis dans les recherches sur le SOPK. De même, le cactus velu est une référence directe à Harnaam Kaur, une jeune activiste londonienne atteinte du syndrome. Diagnostiquée dès son plus jeune âge comme étant atteinte du SOPK, elle a longtemps souffert d'être stigmatisée en raison de sa pilosité très intense. Ayant d'abord tenté de lutter contre ce symptôme de la maladie lié au débalancement hormonal, elle opère au début de la vingtaine un changement de cap radical et décide d'embrasser sa condition et d'en faire un atout. Depuis, elle arbore fièrement une longue barbe dense et un torse velu, lesquels contrastent avec un maquillage élaboré, des bijoux et ongles peints. Aujourd'hui modèle professionnel, elle est représentée par deux agences de mannequins - Ugly Rage à Londres et Wanted à Paris - toutes deux consacrées aux profils physiques atypiques.

En reprenant tous les codes de la féminité normative et en les superposant à des attributs masculins, Harnaam Kaur revendique la beauté du hors-norme et le droit à tout un chacun d'être reconnu et valorisé dans sa différence. Dans une série photo célèbre réalisée pour un magazine, Kaur pose vêtue d'une tenue de mariée, sa barbe garnie de fleurs, référence que reprend Stokes dans son œuvre, incrustant un de ses cactus de fleurs en hommage au modèle. En reprenant tous les clichés associés à représentation de la féminité - douceur et couleurs pastels, fleurs et brillants - et en les mettant en parallèle à la figure de l'activiste, l'artiste en fait un plaidoyer pour ces femmes qui doivent 
transiger avec les effets du SOPK. Et finalement, au-delà du discours sur la maladie et ses effets néfastes, elle propose une prise de position féministe célébrant la femme dans sa pluralité et une subversion des idéaux normatifs imposés par la société.

\section{Conclusion}

En s'immisçant dans l'univers des chercheurs, l'art est sans doute à même de participer à démystifier certains enjeux de la bioéthique et surtout de rendre cette recherche - et ses applications possibles vivante et attrayante pour le public. Ainsi, dans certains cas, le travail de l'artiste peut être perçu comme une forme de médiation, mais il ne faut toutefois pas injustement réduire son rôle à celui de simple intermédiaire ou de vulgarisateur scientifique. En effet, l'œuvre demeure surtout un lieu de réflexion privilégié où, via divers dispositifs, mises en scène et stratégies esthétiques, pourra s'opérer un jeu complexe et fécond entre réalité et fiction. Cet acte de modélisation est notamment possible parce que l'art n'est pas réprimé par les contraintes du concret ou de la pure réalité, parce qu'il laisse place à la métaphore, à l'imagination et à la subjectivité. Ainsi, par les nuances, les décalages et les " sensibilités étrangères » [12] qu'il propose, le travail de l'artiste permet d'aiguiser notre jugement et notre manière d'appréhender le réel peut se faire d'un regard quelque peu transformé.

Bien que les rencontres provoquées par le projet et l'exposition Art + Bioéthique aient été pour tous enrichissantes, le spectateur de l'exposition, et ici le lecteur, sera peut-être à même de décoder, au fil des essais et des œuvres les variations de dynamiques d'un binôme à l'autre. Nous souhaitions une relation d'échange, d'inspiration et d'enrichissement mutuel et les résultats laissent parfois transparaître une grande complicité entre les acteurs. Dans certains cas, la " co-contamination » des idées demeure plus discrète, et dans d'autres les échanges auront été riches et fructueux, mais un inconfort voire même un malaise demeure face à la production de l'autre. Bien que les différentes dynamiques observées soient le propre des rencontres interindividuelles, elles témoignent néanmoins tout autant de la rencontre de deux cultures, ainsi que du fossé existant entre les stratégies et les usages spécifiques à chacune. Elles indiquent la pertinence d'entrecroiser ces deux univers et d'exposer la pluralité de conceptions qui peuvent naître autour d'un sujet spécifique. Elles exposent l'aspect sensible des questions qui touchent au vivant, qu'il soit humain ou animal, et l'intérêt d'y apporter une réflexion interdisciplinaire. Et finalement, elles démontrent l'importance des sujets abordés et leur nécessaire mise en débat.

\section{Références}

1. Kinkead A. 54 rue DuBalcon. BioéthiqueOnline. 2016;5/23.

2. Rathwell-Deault D. L'animal un co-citoyen, et pourquoi pas? BioéthiqueOnline. 2016;5/24.

3. Noury M. Na-no-body: De l'oubli du corps sensible en nanomédecine. BioéthiqueOnline. 2016;5/20.

4. Coleman S. Na-no-body. BioéthiqueOnline. 2016;5/19.

5. Le Mée M. Mael-e(s)t-France. BioéthiqueOnline. 2016;5/27.

6. Foucault M. Les anormaux, cours au Collège de France. 1974-75. Paris : Seuil/Gallimard. 1999.

7. Beaudry J-S. The anxious heart of injustice: negative affective responses to disabilities. BioéthiqueOnline. 2016;5/28.

8. Ménard J-F. Cobayes de la relève : l'artiste et le chercheur à la rencontre de l'expérience. BioéthiqueOnline. 2016;5/18.

9. Lavoie-Lachapelle A. Jour de fête! BioéthiqueOnline. 2016;5/17.

10. Doudenkova V. La bioéthique, l'art et le syndrome des ovaires polykystiques: propos impressionniste visant à réhabiliter les corps tabous et les ovaires blâmés. BioéthiqueOnline. 2016;5/22.

11. Stokes G. Constant Beauty Within and PCOS as Cacti. BioéthiqueOnline. 2016;5/21.

12. Elgin CZ. Les fonctions de la fiction. Les Cahiers du Musée national d'art moderne. 1992;41(automne):33-44. 